\title{
Implementación de una unidad didáctica basada en el aprendizaje del inglés para fines específicos en el área de gastronomía y hotelería
}

\begin{abstract}
................................................ Carmen Gloria Garrido Barra' \& Karen Duyvestein Olivares ${ }^{2}$
1. Directora del Programa de Formación Pedagógica, Universidad del Desarrollo, Concepción, Chile;

cggarrido@udd.cl

2. Profesora en Educación Media con mención en Inglés, egresada de la Universidad del Desarrollo, Concepción, Chile;

kduyvestein@gmail.com
\end{abstract}

Recibido: 29 de octubre del 2015 Corregido: 09 de marzo del $2016 \quad$ Aceptado: 30 de marzo del 2016

\begin{abstract}
Resumen
En el mundo globalizado en el que vivimos hoy, aprender una lengua extranjera es prácticamente una obligación, especialmente para aquellas personas que se desenvuelven en el área de la gastronomía y hotelería. La presente intervención tuvo por objetivo contextualizar la enseñanza del inglés en un establecimiento educacional técnico profesional chileno con mención en gastronomía y hotelería, con un grupo de cinco alumnas de segundo medio y cinco alumnas de tercero medio (que corresponden a los dos últimos niveles de la educación secundaria chilena). Para lograr el objetivo, se utilizó un modelo de enseñanza denominado Inglés para Fines Específicos (ESP, por sus siglas en inglés) que se centra en las necesidades de los alumnos y en ayudarlos a cumplir sus propósitos o metas. El uso de este modelo logró no solo incrementar el interés de las alumnas por aprender inglés como lengua extranjera, sino que además les ayudó a descubrir para qué y por qué es tan necesario e importante aprender inglés.
\end{abstract}

Palabras clave: Inglés como lengua extranjera, enseñanza centrada en el alumno, inglés para fines específicos, hotelería, gastronomía.

\section{Abstract \\ Implementation of a unit based on learning english for specific purposes in the area of gastronomy and hotel industry}

In the global world we live in, learning a foreign language is almost an obligation, especially for those who work in the gastronomy and hotel industry. This pedagogical intervention aimed at contextualizing the teaching - learning process of English as a foreign language in a Chilean technical school with a major in gastronomy and hotel industry. Five women from third high School level and five women from fourth high School (these two levels correspond to the last two levels of high school in Chile) level participated in this project. To reach the objective, the unit was organized following ESP principles. ESP focuses on students' needs and on helping them reach their aims. The use of ESP helped students not only to learn English as a foreign language but also to discover why learning English is so important.

Key words: English as a foreign language, student centred teaching, English for specific purposes, gastronomy and hotel industry. 


\section{INTRODUCCIÓN}

Actualmente, todos somos testigos de cómo el inglés se ha convertido en una de las lenguas más poderosas y dominantes del mundo. Aprender inglés pareciera ser la única forma de desarrollarse y progresar tanto personalmente como profesionalmente (Bakhtawar, 2012). Las razones por las que los individuos deciden aprender inglés son variadas: algunas lo estudian porque forma parte del currículo educativo de su país y otros porque tienen algún tipo de propósito específico que desean cumplir mediante el aprendizaje de este idioma (Harmer, 2007). En el caso de las personas ligadas al área de la gastronomía y hotelería, el aprendizaje del inglés se convierte en una necesidad al momento de querer insertarse exitosamente en el mundo laboral. Por consiguiente, el Ministerio de Educación de Chile a contar del año 2004, ha implantado un programa denominado Inglés Abre Puertas cuyo objetivo consiste en intensificar la enseñanza-aprendizaje del idioma inglés con el fin de que los ciudadanos chilenos pueden acceder a mejores oportunidades laborales y académicas.

El presente documento se centrará en describir una intervención didáctica que tuvo por objetivo contextualizar la enseñanza del inglés en un establecimiento educacional técnico profesional chileno con mención en gastronomía y hotelería, de dependencia particular y católico, cuyo sello es el egreso de mujeres líderes en espíritu de servicio, que aporten positivamente en la sociedad todo esto dentro de un clima de libertad, responsabilidad y autonomía, en donde se desarrollan las capacidades necesarias para que las alumnas puedan afrontar los retos que la sociedad plantea a nivel tecnológico y académico, además de sustentar todo en valores cristianos. Este establecimiento permite a sus alumnas obtener el título técnico de la especialidad de hotelería con mención en gastronomía, junto con la Enseñanza Media. Específicamente, se trabajó con los cursos de segundo y tercero medio (que corresponden a los dos últimos niveles de la educación secundaria chilena). Cada curso estaba compuesto por cinco alumnas.

Para lograr el objetivo de la intervención (contextualizar la enseñanza del inglés) se utilizó un modelo de enseñanza denominado Inglés para Fines Específicos (ESP, por sus siglas en inglés) que se centra en las necesidades de la población estudiantil y en ayudarlos a cumplir sus propósitos o metas y se diseñó la unidad May I take your order, please?

Con base en los resultados obtenidos, podemos afirmar que la implementación de unidades didácticas basadas en ESP como la que a continuación se describe, presentan claros beneficios para el aprendizaje de una lengua extranjera. Por una parte, esta metodología ayuda a mejorar el desempeño lingüístico y por otra, aumenta la motivación de los estudiantes por aprender una lengua extranjera.

\section{La corriente humanista y la enseñanza del inglés}

A pesar de que actualmente la población inglesa está en constante contacto con otras culturas tales como la china u hispana (con las que se relaciona por negocios, política, turismo, etc.), el inglés sigue siendo el idioma dominante en el mundo de las comunicaciones, de la gastronomía y hotelería, de los negocios, y de la educación, entre otros. Por tanto, con el fin de cubrir la gran demanda por el aprendizaje del inglés en distintos ámbitos, nace la enseñanza del inglés para fines específicos que pretende contextualizar la ya existente enseñanza del inglés con fines generales (IFG).

La idea de la enseñanza centrada en la población estudiantil y sus necesidades no es nueva. Hutchinson y Waters (2007) señalan que el nacimiento de la corriente humanista emerge tras la enorme expansión sin precedentes de la actividad científica, técnica y económica a escala internacional. Los autores señalan que tras la segunda guerra mundial existían dos corrientes dominantes: el comercio y la tecnología, además, debido al poder económico de Estados Unidos en ese tiempo, fue el inglés el que predominó como lengua general. 
El aumento del número de personas interesadas en aprender inglés para poder entrar en este nuevo mercado hizo que naciera esta nueva forma de enseñar, la que se centraba no solo en la enseñanza de los contenidos lingüísticos, sino que además consideraba en su planificación las necesidades y el contexto en el cual los estudiantes usurarían el idioma.

Carl Rogers (1969) en su obra Freedom to Learn planteaba una nueva visión de enseñanza basada en que los profesores dejaran de centrarse en sí mismos y comenzaran a visualizar las necesidades, metas y/o propósitos de sus propios estudiantes. Esta corriente se denominó enseñanza basada en la población estudiantil y es la base del ESP. La aplicación de técnicas humanísticas es de suma relevancia en la enseñanza de un idioma, ya que apunta directamente a cumplir las metas y propósitos de la población estudiantil. Centrarse en las necesidades de los estudiantes, en ayudarlos a cumplir sus propósitos o metas que son la base de la motivación.

El humanismo, y por tanto la enseñanza del inglés para fines específicos, tienen un número de mensajes para el profesor de idiomas.

Crear un sentimiento de pertenencia, lograr que los temas sean relevantes para el aprendiz, promover el conocimiento de sí mismo, desarrollar una identidad personal, estimular la autoestima, involucrar los sentimientos y las emociones, minimizar la crítica, desarrollar el conocimiento del proceso de aprendizaje, estimular la iniciativa, permitir elecciones y estimular la autoevaluación (Williams y Burden, 2002, p.38).

Las actividades de la sala de clases, por lo tanto, deben utilizar contextos significativos y establecer una comunicación genuina en la cual los estudiantes se vean involucrados como sujeto social.

\section{Inglés para fines específicos}

La característica distintiva de la enseñanza para fines específicos (ESP en inglés) es el hecho de que las clases se planifican con base en las necesidades de los estudiantes. Las preguntas básicas son las siguientes: ¿para qué necesitarán nuestra población estudiantil el idioma? ¿Qué habilidades deben desarrollar? ¿A qué nivel deben ser desarrolladas estas habilidades?

Un aspecto importante por recordar, es que una clase basada en el enfoque ESP tiene como objetivo principal la enseñanza del lenguaje, el discurso y las habilidades comunicativas necesarias para desenvolverse en el idioma extranjero. Por tanto, es necesario unir el enfoque comunicativo del idioma con las necesidades de la población estudiantil.

En el enfoque comunicativo, el objetivo de la enseñanza es desarrollar la competencia comunicativa, es decir, "la habilidad de usar el lenguaje de forma apropiada al contexto, con el propósito de lograr objetivos" (Carter y Nunan, 2002, p.219). En este sentido, el aprendizaje de una lengua extranjera no se reduce al conocimiento de los aspectos organizacionales del sistema, sino que es un proceso entendido como la adquisición de medios lingüísticos para realizar diferentes funciones sociales. En este enfoque, los aprendices de una lengua extranjera necesitan desarrollar un conocimiento sobre cuáles son los propósitos de los actos comunicativos y de cómo lograrlos a través de formas lingüísticas.

Antes de organizar una clase de ESP, debemos recordar que no es necesario que el docente sea un experto en el área de especialización de los estudiantes. Scrivener (2010) sugiere combinar el dominio del idioma del profesor con el dominio del tema de los estudiantes en el momento de planificar una unidad de ESP.

Harding (2007) señala que existen cuatro puntos por considerar para planificar una unidad de ESP. De acuerdo con el autor, lo primero es definir las necesidades de la población estudiantil. Un segundo punto es comprender la naturaleza del área de estudio de los estudiantes. Harding recomienda visitar el 
lugar de trabajo, talleres, laboratorios, etc. Si la población estudiantil aún no están trabajando, sugiere pedirles que se imaginen cómo podrían usar el idioma en su trabajo. En otras palabras, pedir a la población estudiantil una visión de futuro. El tercer punto es destinar tiempo para decidir los contenidos lingüísticos que se enseñarán de acuerdo con el área de trabajo o estudio. Finalmente, el experto sostiene que es necesario usar contextos, textos y situaciones propias del área de estudio o trabajo de la población estudiantil, ya sean reales o simulados. De esta forma, el lenguaje se usará en forma natural.

El inglés para fines específicos es sin duda una valiosa herramienta que permite contextualizar la enseñanza del inglés y entregar las herramientas necesarias para utilizar la lengua extranjera en contextos reales y de interés de la población estudiantil. Por otra parte, y como señalan Hutchinson y Waters (2007), el hecho de que los aprendices sepan específicamente por qué están aprendiendo un idioma ayuda a aumentar la motivación.

\section{METODOLOGÍA}

\section{Diagnóstico}

Durante el primer semestre del año lectivo de 2013, se observaron dos cursos: segundo y tercero medio (en cada curso asistían cinco alumnas), con el fin de detectar las fortalezas y debilidades del establecimiento, docente de inglés y alumnas en relación con el dominio del idioma inglés y el desarrollo de las clases.

Se eligieron estos cursos, pues las alumnas habían manifestado a la dirección de la institución que tenían problemas para comprender el idioma y que además no les agradaba estudiar inglés. En atención a estos comentarios, el colegio decidió realizar un taller que permitiera a las alumnas reforzar contenidos, ayudarlas a desarrollar habilidades de comunicación oral en inglés y sobretodo fomentar su interés por la asignatura.

El taller se realizó una vez a la semana y con una duración de 45 minutos cada clase. Este taller significó una clase extra para las alumnas, puesto que las clases de la asignatura continuaron desarrollándose en forma habitual. Por lo tanto, dado que la dirección buscaba fomentar el interés el interés de las estudiantes por aprender inglés, y no una mejora en las calificaciones de la asignatura, decidió que si bien el taller sería evaluado, las calificaciones no interferirían con las calificaciones de la asignatura. Es decir, la evaluación tendría solo un carácter formativo.

El estudio de la situación consistió en el análisis de la hoja de observación de clases, (ver anexo 1) y la aplicación de un análisis de fortalezas, oportunidades, debilidades y amenazas (FODA), (ver anexo 2). Los instrumentos anteriormente descritos y la evaluación inicial llevaron a la detección de la problemática: descontextualización de la enseñanza del inglés como lengua extranjera.

\section{Diseño e implementación de la unidad didáctica}

Con el fin de contextualizar la enseñanza del inglés se diseñó una unidad didáctica denominada "May I take your order, please?". Esta unidad fue creada basándose en la metodología ESP, es decir, se diseñó con el propósito de realizar trabajos prácticos de manera que las alumnas pudieran recrear situaciones reales presentadas en el área de la gastronomía y la hotelería. 
Cada clase de la unidad fue planificada considerando el objetivo general de la unidad: ordenar y tomar un pedido en un restaurante e incluyó objetivos específicos y actividades graduadas en dificultad. La planificación también incluyó las cuatro evaluaciones que se realizaron: diagnóstica, autoevaluación, evaluación oral de proceso y evaluación del desempeño de la docente.

La implementación de la unidad tuvo una duración de 10 sesiones de 45 minutos cada una y se desarrolló de la siguiente forma:

En la primera clase, se explicó a las alumnas el proyecto y se les presentó la unidad didáctica. Además, se les solicitó crear en parejas un diálogo en el que recrearan la escena de un restaurante. Las siguientes clases, las alumnas presentaron su juego de roles. Esta actividad fue evaluada con una rúbrica y se entregó retroalimentación a cada grupo (ver anexo 3).

Durante las dos clases siguientes, las alumnas aprendieron vocabulario relacionado con alimentos y expresiones típicas de un restaurante. Para trabajar con vocabulario referido a alimentos, se utilizó material concreto, por ejemplo: un carro de supermercado de juguete con frutas, verduras y alimentos en su interior. De esta forma, las alumnas podían manipular el vocabulario aprendido. Además, para complementar esta actividad se desarrollaron guías de trabajo. Estas guías consideraron ejercicios tales como las siguientes: sopas de letras, ejercicios con oraciones incompletas y actividades donde debían unir conceptos. Las alumnas trabajaban de manera individual y que luego se corregían en conjunto para finalizar la clase con una retroalimentación general.

Luego de estas tres semanas, las alumnas tuvieron una evaluación formativa en la que se midió el vocabulario aprendido hasta ese momento. Esta evaluación incluyó ejercicios con oraciones incompletas, ejercicios donde debían unir conceptos e identificar imágenes y preguntas de desarrollo. Las estudiantes analizaron distintos tipos de menús y sus respectivas especialidades. Luego en una guía de trabajo, las alumnas identificaron las distintas partes de un menú y su organización.

La siguiente clase (número 6), las estudiantes en dos grupos, tuvieron la oportunidad de crear su propio menú. Para esto se les facilitó materiales tales como goma, cartulina de colores, hojas de block, papel holográfico, tijeras y lápices de colores. De esta forma, las alumnas confeccionaron sus menús y presentaron al resto del curso su restaurante y la especialidad de la casa. Una vez finalizada la clase, las alumnas realizaron una autoevaluación con el propósito de reflexionar y evaluaron su propio desempeño en la actividad realizada en clases (ver anexo 4).

En la clase siguiente (número 7), las alumnas vieron un video en el que se mostraba el protocolo a seguir en el momento de ir a un restaurante. Luego, se les entregó una guía de trabajo en el que se les explicaba cuáles eran los diferentes actores en un restaurante, sus roles y estructuras gramaticales típicas utilizadas en este contexto. Esta información sirvió de referencia para desarrollar su trabajo final.

Las dos clases siguientes, se dedicaron a la confección y organización de la presentación final, se realizaron correcciones y se practicó pronunciación.

Finalmente, en la última clase, las alumnas presentaron en conjunto su trabajo final. Para esto, ambientaron la entrada del colegio como un restaurante. Con tiempo le presentaron a la profesora el tema del restaurante y la especialidad: pizza. El día de la presentación, las alumnas llevaron una pizza y bebestibles. Esta presentación fue grabada y evaluada con una rúbrica que incluyó vocabulario, fluidez, gramática, creatividad, duración y los principales puntos de conversación en la situación de ordenar y tomar un pedido en un restaurante. Una vez finalizada esta actividad las alumnas evaluaron el desempeño de la profesora durante este proceso (ver anexo 6). 


\section{RESULTADOS}

De acuerdo con lo observado en la etapa de diagnóstico, las alumnas no veían la utilidad al aprendizaje del inglés. Las estudiantes sabían que aprender un idioma era bueno para su futuro y que debían hacerlo, pero no tenían claro para qué.

Durante el desarrollo de la innovación didáctica, las alumnas tuvieron cuatro instancias de evaluación. Estas evaluaciones tuvieron como objetivo detectar conocimientos previos, dar a las alumnas la oportunidad de juzgar su propio desempeño, evaluar la capacidad de atender correctamente a clientes extranjeros en un restaurante y finalmente evaluar el desempeño de la docente.

La primera evaluación tuvo como fin de evaluar los conocimientos previos de las alumnas con respecto al tema de la unidad. Con el propósito de hacer la evaluación oral más objetiva, se utilizó una rúbrica que medía aspectos específicos y no solo una impresión general. Los aspectos evaluados fueron el uso de los principales puntos de conversación dados en situación de restaurante, pronunciación, comprensión del mensaje y el orden de las estructuras gramaticales utilizadas en inglés.

De las cinco parejas evaluadas, ninguna logró utilizar los principales puntos de conversación establecidos para la ocasión. Las alumnas solo utilizaron los puntos más básicos: bienvenida al restaurante, entrega de menú, ordenar bebestible, ordenar comida, pedir cuenta y agradecer a la mesera. Con respecto a la pronunciación y claridad del mensaje ninguna pareja obtuvo el $100 \%$ del puntaje asignado, ya que constantemente se reían o dudaban al hablar lo que las hacía repetir su diálogo, probablemente por nerviosismo e inseguridad. Además, dos de las cinco parejas, utilizaron vocabulario erróneo lo que dificultó aún más la comprensión del mensaje final. Por otra parte, en cuanto al uso de las estructuras gramaticales no hubo mayor problema. El $80 \%$ de las alumnas lograban realizar oraciones compuestas por sujeto, verbo y predicado, incluso utilizaban correctamente los verbos. Algunos ejemplos de estas oraciones son: May I take your order, please?, What would you like to eat?, Welcome to our Restaurant, I would like to meet the chef.

TABLA 1

Resultados evaluación inicial

\begin{tabular}{lccccc}
\multicolumn{1}{c}{ Criterio de evaluación } & Pareja 1 & Pareja 2 & Pareja 3 & Pareja 4 & Pareja 5 \\
\hline $\begin{array}{l}\text { Las alumnas utilizan los principales puntos de } \\
\text { conversación. }\end{array}$ & $0 / 5$ & $0 / 5$ & $0 / 5$ & $0 / 5$ & $0 / 5$ \\
\hline $\begin{array}{l}\text { La pronunciación es clara y el mensaje } \\
\text { se entiende con facilidad. }\end{array}$ & $2 / 5$ & $2 / 5$ & $3 / 5$ & $3 / 5$ & $2 / 5$ \\
\hline $\begin{array}{l}\text { Las alumnas utilizan correctamente estructuras } \\
\text { gramaticales básicas. Los errores no perjudican } \\
\text { la comprensión del mensaje. }\end{array}$ & $3 / 5$ & $2 / 5$ & $3 / 5$ & $4 / 5$ & $3 / 5$
\end{tabular}

Fuente: elaboración propia.

Como se puede observar en la tabla 1, los resultados confirmaron que las estudiantes no estaban en condiciones de ordenar y tomar un pedido en un restaurante, además, dejó de manifiesto la necesidad de adecuar las actividades de manera que se disminuyera la ansiedad y fomentara la seguridad en las estudiantes.

La segunda evaluación fue una autoevaluación. El objetivo fue dar la oportunidad a las estudiantes de juzgar su propio desempeño en la actividad relacionada con la creación de un menú y la presentación de este al resto de las compañeras. 
Las alumnas debían marcar con una "X" la opción que más se asemejara a su realidad. El día de la autoevaluación, se encontraban presente solo ocho alumnas. En esta autoevaluación, el $100 \%$ de las alumnas presentes manifestaron agrado por la actividad. Todas señalaron haber cooperado y escuchado a sus compañeras durante el desarrollo del trabajo grupal. Sin embargo, solo un 50\% manifestó haber participado activamente en clases.

TABLA 2

Resultados autoevaluación

\begin{tabular}{|c|c|c|c|}
\hline Criterio de evaluación & & & \\
\hline Me gustó la actividad & & & $8 / 8$ \\
\hline Cooperé con el grupo durante el desarrollo de la actividad & & $2 / 8$ & $6 / 8$ \\
\hline Participé en clases & $3 / 8$ & $1 / 8$ & $4 / 8$ \\
\hline Escuché atentamente a mis compañeras y profesora & & $3 / 8$ & $5 / 8$ \\
\hline
\end{tabular}

Fuente: elaboración propia.

Como se puede observar en la tabla 2, esta autoevaluación también ayudó al desarrollo de la autonomía de las alumnas al pedirles que juzgaran su propio desempeño y se dieran cuenta de la importancia de practicar el idioma para obtener mejores resultados.

La tercera evaluación consistió en una evaluación oral de proceso. El objetivo fue evaluar la capacidad de las alumnas de atender correctamente a clientes extranjeros en un restaurante. Los criterios en esta evaluación fueron similares a aquellos utilizados en la evaluación inicial para así poder contrastar resultados y medir el progreso de las alumnas. Para esta evaluación las estudiantes decidieron trabajar todas juntas. Los roles contemplados para la presentación eran: clientes (cinco alumnas), anfitriona (una alumna), gerente (una alumna), meseras (dos alumnas), chef principal (una alumna). El día de la evaluación solo nueve alumnas se encontraban presentes, ya que una se encontraba enferma. Las alumnas solo practicaron una vez antes de la grabación final. Esta evaluación fue grabada y analizada en conjunto para la correspondiente retroalimentación. Los resultados de la evaluación fluctuaron entre los criterios de evaluación "excelente" y "bueno".

TABLA 3

Resultados evaluación oral de proceso

\begin{tabular}{|c|c|c|}
\hline Criterio de evaluación & Excelente & Bueno Satisfactorio Insatisfactorio \\
\hline Vocabulario & $\mathrm{X}$ & \\
\hline Fluidez & $\mathrm{x}$ & \\
\hline Gramática & & $x$ \\
\hline Creatividad & $x$ & \\
\hline Tiempo & $x$ & \\
\hline $\begin{array}{l}\text { Principales puntos de conversación: bienvenida y } \\
\text { ubicación, entregar menú y especialidades, ordenar } \\
\text { bebestibles, discutir que se desea ordenar, ordenar, hacer } \\
\text { comentarios sobre la comida, ordenar el postre, pedir la } \\
\text { cuenta, comentar y deja propina, agradecer y despedirse. }\end{array}$ & & $\mathrm{X}$ \\
\hline
\end{tabular}

Fuente: elaboración propia. 
Los resultados de esta evaluación muestran un claro avance de las estudiantes. En relación con los aspectos lingüísticos se destacan la fluidez y vocabulario. La gramática y principales puntos de conversación se encuentran dentro del rango bueno, probablemente con más ejercitación se podría lograr el 100 $\%$ de las estudiantes dentro del rango excelente. El $100 \%$ de logro de todas las alumnas en los aspectos creatividad y duración demuestran el alto compromiso de las estudiantes con la actividad.

Finalmente, las alumnas evaluaron el desempeño de la docente. Esta evaluación se realizó una vez finalizada la última clase. Las alumnas se llevaron la evaluación y debían dejarla en la sala el lunes siguiente. Esta evaluación tuvo como objetivo que las alumnas evaluaran el desempeño de la profesora con el fin de entregar retroalimentación de su práctica docente. Todas las alumnas entregaron la evaluación a tiempo. Los resultados muestran que el $100 \%$ de las alumnas estaban conformes con el desempeño de la profesora durante los talleres de inglés oral. Solo dos alumnas señalaron tener problemas con la comprensión del inglés durante las clases.

TABLA 4

Resultados evaluación desempeño docente

\begin{tabular}{lccc|}
\hline \multicolumn{1}{c}{ Criterio de evaluación } & Siempre & A veces & Nunca \\
\hline Contenidos & $100 \%$ & \\
\hline Didáctica & $100 \%$ & \\
Comprensión & $80 \%$ & $20 \%$ \\
Puntualidad & $100 \%$ & \\
Cercanía con los alumnos & $100 \%$ &
\end{tabular}

Fuente: elaboración propia.

La favorable evaluación obtenida por la profesora demuestra que las alumnas valoraron el compromiso de la docente y que el diseño de la unidad fue el apropiado. La dificultad de dos alumnas en comprender mensajes en inglés, probablemente se habría superado con más horas de exposición al idioma.

\section{DISCUSIÓN DE RESULTADOS}

De las observaciones realizadas durante el primer semestre lectivo, se concluyó que las alumnas no veían los beneficios de aprender inglés asociados a su área de estudio. Por lo anterior, se decidió que el enfoque de la enseñanza del inglés como lengua extranjera en un establecimiento técnico-profesional debía ser específico, orientado el área de especialización de las alumnas. En otras palabras, se debía utilizar la metodología ESP. De esta forma, las alumnas tendrían la oportunidad de ver en contexto la utilidad de aprender inglés como lengua extranjera.

La motivación debe orientarse a conseguir su activación en dos direcciones: interés para hacer lo que les gusta y voluntad para hacer lo que no les gusta. Las dianas del profesorado deberían ser despertar el interés (seleccionando contenidos interesantes podando contenidos superfluos para que quepan los temas que les entusiasmen) y fortalecer la voluntad (con superación de obstáculos y debilidades) (Vaello, 2013, pp. 1-2).

De acuerdo con los resultados obtenidos en la evaluación inicial, las alumnas, efectivamente, presentaban problemas de vocabulario y de uso de estructuras comunicativas típicas utilizadas en un restaurante. Las alumnas confundían palabras y en vez de utilizar la palabra "menu" utilizaban la palabra "letter" para referirse a una carta o menú en el que se presentan las comidas o platos en un restaurante. Además, al presentar su diálogo, las alumnas se reían constantemente, lo que ponía en evidencia su nerviosismo 
e inseguridad. Para trabajar el léxico se diseñaron hojas de trabajos con vocabulario específico y se trabajó con material concreto. Para disminuir la ansiedad de las estudiantes en el momento de hablar, se optó por el trabajo en grupo y actividades de carácter lúdico.

Durante el proceso, las alumnas se autoevaluaron. En esta autoevaluación, ellas señalaron que les agradaba la actividad relacionada con la creación de un menú. También indicaron haber cooperado con su grupo durante el desarrollo de la actividad. Lo anterior, demuestra que considerar los intereses de los estudiantes, ayuda a fomentar la participación y motivación de los estudiantes. La participación es un punto relevante, si consideramos que un idioma extranjero solo puede ser aprendido si se practica. Por otra parte, la autoevaluación ayudó a las estudiantes a monitorear su aprendizaje y así convertirse en aprendices más autónomas.

Al comparar la evaluación inicial con la evaluación final podemos concluir que el rendimiento de las alumnas mejoró significativamente: utilizaron correctamente estructuras gramaticales y vocabulario aprendido durante la innovación. La presentación fue más fluida y ellas estaban más seguras de sí mismas y de su desempeño. Solo bastó una práctica previa a la grabación final. Las alumnas no se reían tanto y ellas no sentían vergüenza al hablar en inglés.

En relación con la evaluación de la docente, se puede apreciar que las alumnas estaban conformes con el desempeño de la docente. Solo dos alumnas señalaron tener problemas con la comprensión del inglés durante las clases. Esto se puede explicar porque las clases se realizaron en su totalidad en la lengua extranjera.

\section{CONCLUSIONES}

La presente intervención tuvo por objetivo contextualizar la enseñanza del inglés como lengua extranjera con el fin de que 10 alumnas de un establecimiento técnico profesional chileno con mención en hotelería y gastronomía lograran aprendizajes significativos. Las alumnas asistieron durante 10 clases de 45 minutos cada una a un taller de reforzamiento en el que se les brindó las herramientas para lograr este objetivo.

Durante el desarrollo de la unidad, se aplicaron cuatro evaluaciones. Cada una con un propósito específico: detectar conocimientos previos, dar la oportunidad a las alumnas de juzgar su propio desempeño y convertirse en aprendices más autónomas, evaluar la capacidad de atender correctamente a clientes extranjeros en un restaurante y finalmente evaluar el desempeño de la docente. El seguimiento del desempeño de las alumnas con tres instrumentos ayudó al monitoreo del proceso de enseñanzaaprendizaje. La positiva evaluación de la profesora confirma la necesidad de que los docentes diseñen unidades considerando las características y necesidades de los estudiantes. En este caso en particular, no solo se consideró los intereses de las alumnas, sino también la variable afectiva. Para este último punto, se diseñaron actividades lúdicas que permitieron reducir el nivel de ansiedad en las estudiantes.

Si bien esta innovación no contempló mejora en las calificaciones por decisión de la dirección del centro educativo, la disposición de las alumnas frente a las clases de inglés era distinta no solo en el taller, sino que en las clases con la profesora de asignatura también: las alumnas participaban más y se atrevían a hablar en inglés. Además, por iniciativa propia las alumnas invitaron a otras profesoras de asignatura de la institución y apoderadas, de tal manera que presenciaran su presentación final, lo que demuestra que ya no sentían vergüenza de hablar inglés frente a otras personas.

La mayor dificultad de la implementación de la unidad didáctica May I take your order, please? fue el tiempo destinado a esta. Contar con solo 45 minutos a la semana para realizar clases limitó los contenidos lingüísticos por enseñar. Sin embargo, la motivación y compromiso de las alumnas permitió el desarrollo 
de la unidad sin inconvenientes. Considerar los intereses y necesidades de las alumnas fue un punto clave en el éxito de esta innovación didáctica. Las alumnas señalaban fuera y dentro del aula que los talleres eran muy entretenidos y didácticos. Esto porque se realizaban actividades lúdicas, juegos, actividades en grupo y manualidades. En general, actividades que salían de la rutina que ellas vivían en las distintas asignaturas. De acuerdo con las evaluaciones las alumnas disfrutaban las clases y ya no sentían que no sabían nada ni que nunca podrían hablar inglés.

Un aspecto importante para lograr la motivación y compromiso de las estudiantes fue lograr que las alumnas tuvieran claridad de la necesidad de dominar el inglés para desenvolverse exitosamente en su futuro campo laboral. Esto se logró a través de conversaciones y de un video utilizado como parte de la clase número 7. Asimismo, podemos decir que un aspecto importante es recordar la necesidad de contextualizar la enseñanza del idioma y de explicitar a los estudiantes la importancia de aprender inglés, especialmente en el área de hotelería y gastronomía.

En esta oportunidad no se consideró el uso de la infraestructura del centro educativo técnico profesional, sin embargo, se sugiere hacerlo en un futuro, puesto que un acercamiento al mundo laboral facilitaría aún más la comprensión por parte de las estudiantes sobre la importancia de aprender inglés, especialmente en su área de estudio y futuro desempeño laboral.

Finalmente, cabe preguntarse ¿Cuáles hubiesen sido los resultados si se hubiese tenido el tiempo necesario para diseñar en conjunto actividades con las alumnas y si se hubiese utilizado la infraestructura con las que ellas cuentan en el establecimiento técnico profesional donde estudian? ¿Hubiesen estado aún más motivadas?

El área de la hotelería es inmensa, es por esto que esta intervención sirve como punto de partida para aquellas personas que quieran indagar más sobre el tema y desarrollar unidades relacionadas con la recepción de clientes, manejo de situaciones problemáticas en un hotel, etc. y logren desarrollar las habilidades lingüísticas en una lengua extranjera que permitan a los profesionales enfrentar exitosamente el mundo globalizado en el que vivimos hoy.

\section{REFERENCIAS}

Bakhtawar, S. (2012). English: The need of today's world. DJ The Dawn Journal. 1 (2), 194-197.

Brown, D. (1994). Principles of Language Learning and Teaching. New York: Pearson Education.

Carter, R. \& Nunan, D. (Eds.). (2001). The Cambridge Guide to Teaching English to Speakers of Other Languages. Cambridge: Cambridge University Press.

Harding, K. (2007). English for Specific Purposes. Oxford: Oxford University Press.

Harmer, J. (2007). How to teach English. Essex: Person Education Limited.

Hutchinson, T. \& Waters, A. (2007). English for Specific Purposes. Cambridge: Cambridge University Press.

Rogers, C. (1969). Freedom to Learn. Columbus: Merril Publishing Co.

Scrivner, J. (2010). Learning Teaching. Oxford: MacMillan.

Vaello, J. (2013). Motivar a los adolescentes. Aula de Secundaria. 1(7), 7-8.

Williams, M. \& Burden, R. (2002). Psychology for Language Teachers. Cambridge: Cambridge University Press. 


\section{ANEXOS}

\section{Anexo 1. Hoja de Observación de Clases}

$\mathrm{UMBC}$

A N HONORS UNIVERSITY I N M A RYLAND

ELC 688: Teaching English to Young Learners

Curso / edad, nivel de Inglés

1. Número de estudiantes en la clase (número de hombres y de mujeres).

2. Contenido y objetivo de la clase observada.

3. ¿La profesora tiene su propia sala o debe compartirla con los demás profesores de asignaturas?

4. ¿Cómo está organizada la sala, las sillas, los muebles, etc.? ¿Qué hay en las paredes de la sala? ¿Qué otros materiales y objetos hay en la sala de clases?

5. ¿Cuáles son los objetivos de la clase? ¿Están estos objetivos relacionados con lenguaje o contenido o ambos?

6. ¿Cómo comienza la clase?

7. ¿Cómo se introduce el vocabulario nuevo? ¿Cómo se presentan estructuras del lenguaje nuevas?

8. ¿De qué forma las alumnas practican inglés?

9. ¿Las alumnas tienen la posibilidad de escuchar, hablar, leer y escribir en inglés?

10. ¿Qué técnicas utiliza la profesora para que las alumnas logren entender lo que se les está enseñando?

11. ¿Qué tipo de interacción se lleva a cabo en la sala de clases? ¿De qué manera participan las alumnas en la clase?

12. ¿Cómo la profesora corrobora el progreso de los estudiantes y el cumplimiento de los objetivos?

13. ¿De qué manera la profesora les entrega retroalimentación a las alumnas?

14. ¿Cómo finalizan las clases? ¿La profesora, asigna tareas o actividades complementarias? 


\section{FORTALEZAS}

Alumnas tienen mucha afinidad y cercanía con sus profesoras.

Existe un alto nivel de compañerismo entre las estudiantes de un mismo curso y entre ambos cursos (2ndo y 3ro Medio).

Excelente trabajo en equipo.

Excelente nivel disciplinar.

Alumnas integras.

No más de 10-12 alumnas por curso.

\section{DEBILIDADES}

Falta de interés de las alumnas por aprender inglés. No tienen claridad sobre la utilidad del inglés en su ámbito profesional.

Falta de confianza en sus conocimientos y habilidades. Falta de compromiso del docente de la asignatura.

\section{OPORTUNIDADES}

Niñas pasan riguroso proceso de selección para ser admitidas en el establecimiento: buen nivel. Colegio financiado por institución externa. Ubicación céntrica del establecimiento Existen comodidades necesarias para que las alumnas desarrollen de mejor manera sus estudios. Libertad de ejercicio docente.

\section{AMENAZAS}

Falta de práctica del inglés hablado. Ubicación céntrica: niñas prefieren salir. Solo dos horas de inglés a la semana. Currículum poco atractivo.

Poca coherencia entre lo que se enseña y lo que necesitan las alumnas.

Al ser internado, los padres se desligan de algunas responsabilidades y éstas recaen en los docentes, en las instructoras o en el cuerpo directivo.

Fuente: elaboración propia.

\section{Anexo 3. Evaluación inicial}

\begin{tabular}{|c|c|c|c|c|c|c|c|}
\hline COMMUNICATION & 0 & 1 & 2 & 3 & 4 & 5 & Total \\
\hline Student uses the main points of conversation. & & & & & & & \\
\hline Pronunciation is clear and makes message easy to understand. & & & & & & & \\
\hline Student makes correct use of grammar structures. Errors do not interfere. & & & & & & & \\
\hline
\end{tabular}

Fuente: elaboración propia.

\section{Main Points of Conversation}

Welcomed and seated Given menu and specialties

Order beverages Comment on food

Thank waiter and leave Discuss and leave tip

\section{Discuss what you want to order}

Order meal

Ask for dessert menu and order dessert Ask for bill

Fuente: elaboración propia. 


\section{Anexo 4. Autoevaluación}

Instructions: check the image that best identifies you.

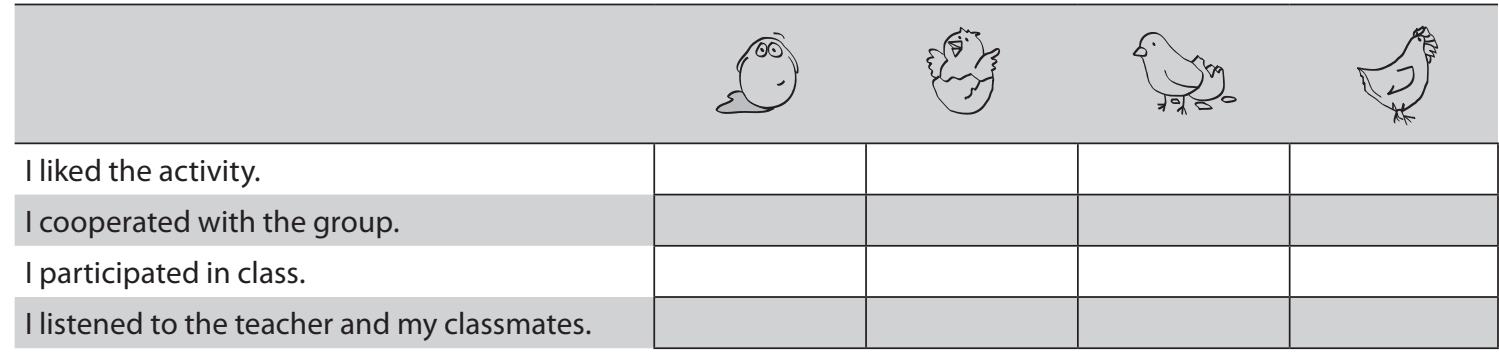

Fuente: elaboración propia.

\section{Anexo 5. Evaluación Final}

\begin{tabular}{|c|c|c|c|c|}
\hline Criteria & $\begin{array}{l}\text { excellent } \\
\text { ( } 5 \text { points) }\end{array}$ & $\begin{array}{l}\text { good } \\
\text { (4 points) }\end{array}$ & $\begin{array}{l}\text { satisfactory } \\
\text { (3 points) }\end{array}$ & $\begin{array}{l}\text { unsatisfactory } \\
\text { ( } 2 \text { points) }\end{array}$ \\
\hline Vocabulary & $\begin{array}{l}\text { A lot of words and } \\
\text { phrases studied in class } \\
\text { are used. }\end{array}$ & $\begin{array}{l}\text { Many words and } \\
\text { phrases studied in class } \\
\text { are used. }\end{array}$ & $\begin{array}{l}\text { Some words and phrases } \\
\text { studied in class are used. }\end{array}$ & $\begin{array}{l}\text { Few words and phrases studied } \\
\text { in class are used. }\end{array}$ \\
\hline Fluency & $\begin{array}{l}\text { No hesitation while } \\
\text { speaking. Assignment } \\
\text { shows good preparation. }\end{array}$ & $\begin{array}{l}\text { Some hesitation while } \\
\text { speaking. Assignment } \\
\text { shows some } \\
\text { preparation. }\end{array}$ & $\begin{array}{l}\text { Hesitations when } \\
\text { speaking. Needs more } \\
\text { preparation. }\end{array}$ & $\begin{array}{l}\text { The script was not well known } \\
\text { and presentation appeared to } \\
\text { be read from notecards. }\end{array}$ \\
\hline Grammar & $\begin{array}{l}\text { Consistent correct use of } \\
\text { grammar and targeted } \\
\text { structures studied in } \\
\text { class. }\end{array}$ & $\begin{array}{l}\text { Occasional errors in } \\
\text { grammar points stud- } \\
\text { ied in class that do not } \\
\text { distract too much from } \\
\text { the task. }\end{array}$ & $\begin{array}{l}\text { Many errors in grammar } \\
\text { points studied in class } \\
\text { that do not distract too } \\
\text { much from the task. }\end{array}$ & $\begin{array}{l}\text { Frequent errors in grammar } \\
\text { points studied in class, inter- } \\
\text { feres with meaning. }\end{array}$ \\
\hline Creativity & $\begin{array}{l}\text { Student shows a lot of } \\
\text { effort and creativity in } \\
\text { adding elements to make } \\
\text { presentation interesting. }\end{array}$ & $\begin{array}{l}\text { Student shows a fair } \\
\text { amount of effort to } \\
\text { add creative elements } \\
\text { to make presentation } \\
\text { interesting. }\end{array}$ & $\begin{array}{l}\text { Student shows some } \\
\text { effort and creativity. }\end{array}$ & $\begin{array}{l}\text { Student shows minimal effort } \\
\text { and creativity and does not do } \\
\text { much to make it interesting. }\end{array}$ \\
\hline Time- limit & Presentation is $5-6^{\prime}$ long & Presentation is $4^{\prime}$ long & Presentation is $3^{\prime}$ long & $\begin{array}{l}\text { Presentation is less than } 3^{\prime} \text { or } \\
\text { more than } 6^{\prime}\end{array}$ \\
\hline $\begin{array}{l}\text { Main points of } \\
\text { conversation }\end{array}$ & It includes all points. & It includes 8-9 points. & $\begin{array}{l}\text { It includes between 5-7 } \\
\text { points. }\end{array}$ & It includes less than 5 points. \\
\hline
\end{tabular}

Fuente: elaboración propia. 


\section{Anexo 6. Evaluación docente}

Instrucciones: de acuerdo a lo observado en clases marque con una cruz la respuesta que más se asemeje a su experiencia vivida en este taller de inglés oral.

\section{Criterios de evaluación}

\section{Siempre A veces Nunca}

Contenidos: La profesora domina los contenidos.

Didáctica: Las actividades realizadas tenían relación con la unidad y eran atractivas.

Comprensión: La profesora explicaba de manera clara y precisa.

Puntualidad: La profesora era puntual al inicio y término de la clase.

Cercanía con los alumnos: La profesora estaba dispuesta a contestar mis dudas o volver a explicar contenidos cuando se le solicitaba.

Fuente: elaboración propia. 